\section{Mortalidade neonatal no Município de Londrina, Paraná, Brasil, nos anos 1994, 1999 e 2002}

\author{
Neonatal mortality in Londrina, Paraná State, \\ Brazil, in 1994, 1999, and 2002
}

\begin{abstract}
Despite technological progress in recent decades, neonatal mortality accounts for some two-thirds of infant deaths where the infant mortality rates are low. This study analyzes neonatal deaths in Londrina, Paraná, Brazil, during three periods, beginning with 1994, the year when pediatric and neonatal intensive care beds were created in the city. The data were collected from live birth certificates in the National Information System on Live Births (SINASC) and individual analysis of neonatal death certificates. Births declined in the city, but the low birthweight rate increased from 7.7 to $8.8 \%$ and the preterm birth rate from 6.3 to $8.4 \%$. Multiple births also increased. Caesarian sections varied from 48 to $52 \%$. The percentage of deaths from congenital malformations increased. The vast majority of neonatal deaths are preventable, mainly by providing specialized care during pregnancy. The neonatal mortality rate has declined recently, from 10.1 to 6.4 per 1,000 live births. The authors conclude that neonatal care is improving in Londrina.
\end{abstract}

Infant Mortality; Birth Weight; Neonatal Intensive Care Units; Pediatric Intensive Care Units
Lígia Silvana Lopes Ferrari ${ }^{1}$ Ângela Sara Jamusse de Brito ${ }^{1}$ Ana Berenice Ribeiro de Carvalho ${ }^{1}$ Maria Rafaela Conde Gonzáles ${ }^{1}$

\section{Introdução}

O coeficiente de mortalidade neonatal é um componente do coeficiente de mortalidade infantil e expressa diretamente a assistência dada ao pré-natal, ao parto e ao período neonatal, refletindo a cobertura e a qualidade dos serviços de saúde de uma região 1,2 .

Em áreas onde o coeficiente de mortalidade infantil pode ser considerado baixo, 60 a $80 \%$ dos óbitos ocorrem em crianças com menos de 28 dias de vida, ou seja, durante o período neonatal 2. Em Londrina, Paraná, Brasil, a porcentagem de óbitos neonatais vem oscilando, desde 1983 , entre 54 a $70 \%$ do total da mortalidade infantil 3 . Houve redução maior do componente pós-neonatal à custa de intervenções específicas em ações básicas de saúde, como, por exemplo: as imunizações; o tratamento adequado de diarréias e infecções respiratórias; o incentivo ao aleitamento materno e a melhoria nas condições de saneamento básico 3. Dessa forma, o componente neonatal passa a ser proporcionalmente maior, e os esforços para redução da mortalidade infantil devem ser concentrados em intervenções para diminuir a mortalidade neonatal.

O conhecimento de algumas variáveis maternas e perinatais pode auxiliar no diagnóstico para o combate à mortalidade neonatal, principalmente em relação às mortes que podem ser evitadas com mudanças de condutas 
assistenciais maternas no pré-natal, durante o parto ou no período neonatal com o aprimoramento dos recursos humanos e tecnológicos. A definição correta das causas de óbito pode auxiliar na organização da assistência perinatal.

Em 1994, ano em que se implantaram as Unidades de Tratamento Intensivo (UTI) Neonatais e Pediátricas na cidade, iniciamos o estudo da mortalidade neonatal no Município de Londrina 4 . Esta pesquisa tem como finalidade descrever os óbitos neonatais e analisar a tendência nos diferentes períodos, a fim de fornecer subsídios aos gestores e profissionais de saúde para o planejamento de programas que atuem na prevenção da mortalidade de recémnascidos.

\section{Material e método}

Foram estudados todos os óbitos neonatais com peso ao nascer igual ou maior de $500 \mathrm{~g}$, de residentes em Londrina, ocorridos no período de 1 de janeiro a 31 de dezembro dos anos de 1994, 1999 e 2002. Foram excluídos, deste estudo, todos os nascimentos e óbitos de não residentes em Londrina.

O Município de Londrina se localiza na região norte do Estado do Paraná, tem, aproximadamente, 450 mil habitantes, $95 \%$ concentrados na área urbana. Ocupa a posição de pólo regional e é a segunda cidade do Estado em termos populacionais.

As informações sobre os nascidos vivos foram obtidas das Declarações de Nascidos Vivos (DNV) utilizadas pelo Sistema de Informação sobre Nascidos Vivos (SINASC), implantado em Londrina em 1993, com busca ativa das DNV nos hospitais e cartórios da cidade. O estudo dos óbitos neonatais foi realizado pelo pesquisador, por meio da análise individualizada de cada Declaração de Óbito (DO). Cabe ressaltar que, nesse município, em situações de causa de óbito mal definida na DO, o Núcleo de Informação em Mortalidade da Secretaria de Saúde (NIM), também implantado em 1993, investiga os prontuários hospitalares ou entrevista o médico responsável ou a família com o objetivo de definir a causa, sendo que o pesquisador participou ativamente de todo esse processo.

Entende-se como causa básica de óbito a doença ou lesão que iniciou a sucessão de eventos mórbidos que levou diretamente à morte (morte natural) ou as circunstâncias do acidente ou violência que produziu a lesão fatal (causas externas) 5. Por exemplo, um recémnascido que foi a óbito por síndrome do desconforto respiratório neonatal devido à prema- turidade, a tentativa é evidenciar a causa que levou ao nascimento prematuro, definida como causa básica do óbito, que, na maioria das vezes, está relacionada a intercorrências na gestação e parto.

As variáveis estudadas foram: tipo de gestação (única ou múltipla); duração da gestação; tipo de parto (vaginal ou cesárea); sexo do recém-nascido; peso ao nascer; causas básicas e associadas dos óbitos neonatais. Considerouse pré-termo todo recém-nascido com idade gestacional inferior a 37 semanas; recém-nascido de baixo peso aquele com peso ao nascer inferior a $2.500 \mathrm{~g}$ e recém-nascido de muito baixo peso se inferior a $1.500 \mathrm{~g}$.

Para o cálculo do coeficiente de mortalidade infantil, utilizou-se a razão entre o número de mortes de menores de um ano e o total de nascidos vivos de mães residentes no município. Para o cálculo do coeficiente de mortalidade neonatal, utilizou-se a razão entre o número de mortes de menores de 28 dias e o total de nascidos vivos no mesmo ano.

Os óbitos foram agrupados segundo critérios de evitabilidade adaptados da Fundação Sistema Estadual de Análise de Dados (Fundação SEADE) 6. Os óbitos foram classificados como reduzíveis por adequado controle da gravidez (P00; P04; P05; P07; P22; P55), adequada atenção ao parto (P02; P03; P08; P10-P15; P20-21), por diagnóstico e tratamento precoces (P23-29; P35-39; P50-61; P70-78), parcialmente reduzíveis (X85Y09; Y10-Y34), não evitáveis (Q) e mal definidos.

As variáveis estão apresentadas em números absolutos e percentuais. A avaliação da tendência das freqüências foi realizada com o teste qui-quadrado para tendência.

\section{Resultados}

O número de nascimentos e os coeficientes de mortalidade infantil e neonatal têm diminuído, conforme os três períodos do estudo (Tabela 1). Houve um decréscimo significativo na tendência da mortalidade infantil no período de estudo ( $p<0,001)$, ocorrendo o mesmo, porém de forma menos acentuada, para a mortalidade neonatal $(p=0,023)$, ressaltando que a diferença significativa ocorre apenas entre 1994 e 2002 (odds ratio - OR = 0,63; intervalo de confiança de 95\% - IC95\%: 0,44-0,91).

Entre 70 a $82 \%$ dos óbitos neonatais ocorreram no período neonatal precoce, ou seja, nos primeiros seis dias de vida, sendo 35 a $42 \%$ nas primeiras 24 horas de vida.

Conforme o tipo de gestação, chama a atenção o fato de que, em 1994, não houve óbito dos 
recém-nascidos de gestação múltipla. No entanto, em 1999 e 2002, as gestações múltiplas contribuíram com o coeficiente de mortalidade neonatal.

A duração da gestação é, sem dúvida, fator muito importante para a sobrevivência dos recém-nascidos. Dos nascidos vivos, $6,3 \%, 6,4 \%$ e $8,4 \%$ foram prematuros (menos de 37 semanas de gestação) em 1994, 1999 e 2002, respectivamente, e há uma relação inversa entre idade gestacional e mortalidade neonatal (Tabela 2).

Nos três anos estudados, o índice de cesariana variou de 48 a 52\%, valor esse muito alto segundo as organizações de saúde.

Com relação ao sexo dos recém-nascidos vivos, houve pequeno predomínio de nascimentos do sexo masculino (51\%) em relação ao feminino (49\%) nos três anos estudados. Em 1999 e 2002, houve predomínio de mortalidade neonatal masculina ( $59 \%$ e $52 \%$, respectivamente).

Analisando o peso ao nascer, verificou-se um índice de recém-nascido de baixo peso (menor que $2.500 \mathrm{~g}$ ) de $7,7 \%, 12 \%$ e 8,8\% em 1994, 1999 e 2002, respectivamente (Tabela 3 ).
A maioria dos óbitos neonatais (65 a 82\%) ocorreu em recém-nascidos de baixo peso (Tabela 3). Em 2002, 50\% dos óbitos ocorreram em recém-nascidos com peso de nascimento inferior a $1.000 \mathrm{~g}$.

Quando se compara 1994 com os outros dois anos do estudo, verifica-se que houve redução do coeficiente de mortalidade neonatal por grupos específicos de peso. Nos recém-nascidos com peso entre 500 e 999g, o coeficiente de mortalidade reduziu de 788 por mil em 1994 para 564 por mil em 2002. Entre os pesos de 1.500 e $2.499 \mathrm{~g}$, o coeficiente de mortalidade neonatal reduziu pela metade quando se comparam os anos de 1994 e 2002.

As causas básicas de óbito (CID-10 5) foram agrupadas em causas perinatais (68 a $76 \%$ ), anomalias congênitas ( 18 a $27 \%$ ) e causas externas (3 a 6\%) (Tabela 4).

Os óbitos por anomalias congênitas ocorreram em ordem decrescente por: malformações múltiplas, cardiopatias, hérnia diafragmática, rins policísticos, hidranencefalia, e outras menos freqüentes.

Tabela 1

Número de nascidos vivos, coeficiente de mortalidade infantil e neonatal, razão de chances e respectivos intervalos de confiança.

\begin{tabular}{|c|c|c|c|c|c|c|c|c|c|}
\hline \multirow{3}{*}{$\begin{array}{l}\text { Ano } \\
1994 \\
1999\end{array}$} & \multirow{3}{*}{$\begin{array}{c}\begin{array}{c}\text { Nascidos } \\
\text { vivos }\end{array} \\
8.315 \\
7.915\end{array}$} & \multirow{3}{*}{$\begin{array}{c}\begin{array}{c}\text { Coeficiente de } \\
\text { mortalidade infantil* }\end{array} \\
17,8 \\
13,3\end{array}$} & \multicolumn{3}{|c|}{ OR (IC95\%) } & \multirow{3}{*}{$\begin{array}{c}\begin{array}{c}\text { Coeficiente de } \\
\text { mortalidade neonatal } * *\end{array} \\
10,1 \\
9,3\end{array}$} & \multicolumn{3}{|c|}{ OR (IC95\%) } \\
\hline & & & 1,00 & \multicolumn{2}{|c|}{ Referência } & & 1,00 & \multicolumn{2}{|c|}{ Referência } \\
\hline & & & 0,74 & 0,58 & 0,95 & & 0,92 & 0,68 & 1,27 \\
\hline 2002 & 6.898 & 10,0 & 0,56 & 0,42 & 0,75 & 6,4 & 0,63 & 0,44 & 0,91 \\
\hline
\end{tabular}

* $\chi^{2}$ trend $=17,03 ; g \cdot I .=1 ; p<0,001$.

$\star * \chi^{2}$ trend $=5,13 ; g . l .=1 ; p=0,023$

Nota: coeficiente de mortalidade infantil e neonatal por mil nascidos vivos.

Tabela 2

Nascidos vivos, óbitos neonatais e coeficiente de mortalidade neonatal segundo a idade gestacional.

\begin{tabular}{|c|c|c|c|c|c|c|c|c|c|c|c|c|}
\hline \multirow[t]{2}{*}{$\begin{array}{l}\text { Idade } \\
\text { gestacional } \\
\text { (semanas) }\end{array}$} & \multirow{2}{*}{\multicolumn{3}{|c|}{$\begin{array}{c}1994 \\
\text { Óbitos } \\
n\end{array}$}} & \multirow{2}{*}{$\begin{array}{c}\text { Coeficiente de } \\
\text { mortalidade } \\
\text { neonatal }\end{array}$} & \multirow{2}{*}{\multicolumn{3}{|c|}{$\begin{array}{c}1999 \\
\text { Óbitos } \\
n\end{array}$}} & \multirow{2}{*}{$\begin{array}{c}\text { Coeficiente de } \\
\text { mortalidade } \\
\text { neonatal }\end{array}$} & \multicolumn{2}{|c|}{$\begin{array}{l}\text { Nascidos } \\
\text { vivos }\end{array}$} & \multirow{2}{*}{$\begin{array}{c}2002 \\
\text { Óbitos } \\
n\end{array}$} & \multirow{2}{*}{$\begin{array}{c}\text { Coeficiente de } \\
\text { mortalidade } \\
\text { neonatal }\end{array}$} \\
\hline & & & & & & & & & $\mathrm{n}$ & $\%$ & & \\
\hline$\leq 27$ & 25 & 0,3 & 21 & 840,0 & 31 & 0,4 & 20 & 645,0 & 26 & 0,4 & 20 & 760,0 \\
\hline $28-36$ & 499 & 6,0 & 37 & 74,0 & 474 & 6,0 & 33 & 70,0 & 552 & 8,0 & 19 & 34,0 \\
\hline $37-41$ & 7.650 & 92,0 & 26 & 3,4 & 7.365 & 93,0 & 21 & 2,9 & 6.280 & 91,0 & 5 & 0,8 \\
\hline$\geq 42$ & 133 & 1,6 & - & - & 36 & 0,5 & - & - & 38 & 0,6 & - & - \\
\hline Ignorado & 8 & 0,1 & - & - & 10 & 0,1 & - & - & 2 & 0,0 & - & - \\
\hline Total & 8.315 & 100,0 & 84 & 10,1 & 7.915 & 100,0 & 74 & 9,3 & 6.898 & 100,0 & 44 & 6,4 \\
\hline
\end{tabular}


Tabela 3

Nascidos vivos, óbitos neonatais e coeficiente de mortalidade neonatal de acordo com o peso ao nascer.

\begin{tabular}{|c|c|c|c|c|c|c|c|c|c|c|c|c|}
\hline \multirow[t]{3}{*}{ Peso (g) } & \multirow{3}{*}{\multicolumn{3}{|c|}{$\begin{array}{c}1994 \\
\text { Óbitos } \\
n\end{array}$}} & \multirow{3}{*}{$\begin{array}{l}\text { Coeficiente de } \\
\text { mortalidade } \\
\text { neonatal }\end{array}$} & \multirow{2}{*}{\multicolumn{2}{|c|}{$\begin{array}{c}\text { Nascidos } \\
\text { vivos }\end{array}$}} & \multirow{3}{*}{$\begin{array}{c}1999 \\
\text { Óbitos } \\
n\end{array}$} & \multirow{3}{*}{$\begin{array}{c}\text { Coeficiente de } \\
\text { mortalidade } \\
\text { neonatal }\end{array}$} & \multirow{2}{*}{\multicolumn{2}{|c|}{$\begin{array}{l}\text { Nascidos } \\
\text { vivos }\end{array}$}} & \multirow{3}{*}{$\begin{array}{c}2002 \\
\text { Óbitos } \\
n\end{array}$} & \multirow{3}{*}{$\begin{array}{c}\text { Coeficiente de } \\
\text { mortalidade } \\
\text { neonatal }\end{array}$} \\
\hline & & & & & & & & & & & & \\
\hline & & & & & $\mathrm{n}$ & $\%$ & & & $\mathrm{n}$ & $\%$ & & \\
\hline $500-999$ & 33 & 0,4 & 26 & 787,9 & 44 & 0,5 & 25 & 568,2 & 39 & 0,6 & 22 & 564,1 \\
\hline $1.000-1.499$ & 52 & 0,6 & 13 & 250,0 & 171 & 2,1 & 11 & 64,3 & 56 & 0,8 & 6 & 107,1 \\
\hline$<1.500$ & 85 & & 39 & 458,8 & 215 & & 36 & 167,4 & 95 & & 28 & 294,7 \\
\hline $1.500-2.499$ & 553 & 6,7 & 16 & 28,9 & 741 & 9,4 & 12 & 16,2 & 512 & 7,4 & 8 & 15,6 \\
\hline$<2.500$ & 638 & & 55 & 86,2 & 956 & & 48 & 50,2 & 607 & & 36 & 59,3 \\
\hline $2.500-2.999$ & 1.847 & 22,0 & 12 & 6,5 & 2.393 & 30,0 & 12 & 5,0 & 1.627 & 23,5 & 5 & 3,1 \\
\hline $3.000-3.999$ & 5.388 & 65,0 & 15 & 2,8 & 4.168 & 3,0 & 13 & 3,1 & 4.339 & 63,0 & 3 & 0,7 \\
\hline$\geq 4.000$ & 423 & 5,1 & & & 398 & 5,0 & 1 & 2,5 & 325 & 4,7 & & \\
\hline$\geq 2.500$ & 7.658 & & 27 & 3,5 & 6.959 & & 26 & 3,7 & 6.291 & & 8 & 1,3 \\
\hline Ignorado & 19 & 0,2 & 2 & & & & & & & & & \\
\hline Total & 8.315 & 100,0 & 84 & 10,1 & 7.915 & 100,0 & 74 & 9,3 & 6.898 & 100,0 & 44 & 6,4 \\
\hline
\end{tabular}

Tabela 4

Óbitos neonatais de acordo com grupos de causas e causas perinatais (CID-10 6).

\begin{tabular}{|c|c|c|c|c|c|c|}
\hline \multirow[t]{2}{*}{ Causas } & \multicolumn{2}{|c|}{1994} & \multicolumn{2}{|c|}{1999} & \multicolumn{2}{|c|}{2002} \\
\hline & $\mathrm{n}$ & $\%$ & $\mathrm{n}$ & $\%$ & $\mathrm{n}$ & $\%$ \\
\hline Perinatais & 64 & 76,0 & 54 & 73,0 & 30 & 68,0 \\
\hline Feto e recém-nascido afetados por fatores maternos (P00) & 6 & 9,4 & 15 & 28,0 & 7 & 23,0 \\
\hline Feto e recém-nascido afetados por complicações da gravidez (P01) & 12 & 19,0 & 13 & 24,0 & 11 & 37,0 \\
\hline Feto e recém-nascido afetados por complicações da placenta e cordão umbilical (P02) & 3 & 4,7 & 6 & 11,0 & 2 & 6,7 \\
\hline Feto e recém-nascido afetados por complicações do trabalho de parto (P03) & 2 & 3,1 & 2 & 3,7 & 1 & 3,3 \\
\hline Transtornos relacionados com duração da gestação (P05-P08) & 18 & 28,0 & 6 & 11,1 & 6 & 20,0 \\
\hline Transtornos respiratórios e cardiovasculares (P20-P29) & 8 & 12,5 & 9 & 16,6 & 2 & 6,7 \\
\hline Infecções específicas perinatais (P35-P39) & 9 & 14,0 & & & & \\
\hline Transtornos hematológicos (P50-P61) & 2 & 3,1 & 2 & 3,7 & 1 & 3,3 \\
\hline Transtornos endócrinos e metabólicos (P70-P74) & 1 & 1,5 & - & & & \\
\hline Transtornos sistema digestivo (P75-P78) & 1 & 1,5 & 1 & 1,8 & & \\
\hline Não especificadas (P96.9) & 2 & 3,1 & - & & & \\
\hline Anomalias congênitas & 15 & 18,0 & 18 & 24,0 & 12 & 27,0 \\
\hline Outras/Externas & 5 & 6,0 & 2 & 3,0 & 2 & 5,0 \\
\hline Total & 84 & 100,0 & 74 & 100,0 & 44 & 100,0 \\
\hline
\end{tabular}

Analisando os óbitos por causas perinatais (Tabela 4), verifica-se que, em 1994, a maior proporção foi definida como prematuridade. Entretanto, em 1999 e 2002, com investigação mais detalhada dos óbitos, o evento desencadeante da prematuridade foi identificado na maioria das vezes; portanto, a causa básica foi definida em causas maternas ou complicações da gestação. Dentre as causas básicas princi- pais, estão a rotura prematura de membranas, hipertensão e infecção urinária materna, geralmente determinando o parto prematuro.

As causas externas são variáveis, ou seja, não se pode prever quais fatores externos vão ocorrer; e, neste estudo, esses foram ocasionados por aspiração acidental de leite, intoxicação exógena materna, morte súbita, interrupção da gravidez com misoprostol e negligência 
criminosa (crianças abandonadas em via pública).

Utilizando os critérios de evitabilidade dos óbitos neonatais divulgados pela Fundação SEADE 6, mais de 70\% dos óbitos neonatais em Londrina poderiam ter sido evitados por adequado controle da gravidez, atenção ao parto, ações de prevenção, diagnóstico e tratamento precoces. Entretanto, a proporção de óbitos não evitáveis está aumentando, pois, em 1994, correspondia a 17,8\%; 24,3\% em 1999 e 27,2\% em 2002 (Tabela 5).

\section{Discussão}

Em Londrina, houve queda expressiva da mortalidade infantil nas últimas décadas, atribuída, principalmente, à expansão e melhoria das ações básicas de saúde 3 . Entretanto, emergiu a importância das causas perinatais nos óbitos infantis.

O coeficiente de mortalidade infantil declinou de 17,8 por mil nascidos vivos em 1994 para 10 por mil nascidos vivos em 2002; valor bastante diferente de 25 por mil nascidos vivos, observado no país como um todo em 20027. Entretanto, se compararmos com coeficientes de mortalidade infantil de alguns países da América Latina e Caribe, em anos próximos a 2000, como, por exemplo, Costa Rica (14\%o), Jamaica (11\%o), Cuba (7\%o), e países desenvolvidos como Estados Unidos (6,8\%o), Canadá (5\%o) e Japão(3\%o), verifica-se que o Município de Londrina apresenta coeficiente elevado ${ }^{8}$.

$O$ coeficiente de mortalidade neonatal (menores de 28 dias) foi de 10,$1 ; 9,3$ e 6,4 por mil nascidos vivos; e os óbitos neonatais representaram $56,7 \%, 70 \%$ e $64 \%$ da mortalidade infantil, respectivamente, nos anos de 1994, 1999 e
2002, mostrando o predomínio do componente neonatal nos óbitos de menores de um ano em Londrina. Analisando numericamente os óbitos neonatais em Londrina, ou seja, 84 óbitos em 1994, 74 em 1999 e 44 óbitos em 2002, parecem números pequenos e diminuíram acentuadamente em menos de dez anos, mas, em 2002, por exemplo, foram 44 crianças que morreram antes de completar um mês de vida, e muitas dessas, por causas consideradas evitáveis. Esse número de óbitos relacionado a fatores perinatais pode até ser maior se levarmos em conta que parte da mortalidade infantil tardia se deve também a problemas neonatais que terminaram em óbitos tardios, após uma seqüência de intercorrências e hospitalizações 9 .

Vários estudos destacam fatores de risco relacionados aos óbitos neonatais, como a não realização de pré-natal, mães adolescentes e baixo peso ao nascer 10,11. Portanto, em áreas onde a população recebe um bom atendimento desde o pré-natal e que se estende ao parto e ao recém-nascido, a mortalidade neonatal será baixa. Entretanto, pode-se verificar que, mesmo em países desenvolvidos, onde a mortalidade neonatal é muito baixa, existe um número de óbitos neonatais inevitáveis que, em geral, são devido a anomalias congênitas complexas e muito graves e a peso muito baixo ao nascimento 12 .

Outro fator que pode contribuir para o aumento da mortalidade neonatal são os nascimentos de gestações múltiplas, pois mais de $50 \%$ dos gêmeos e mais de $90 \%$ dos trigêmeos são recém-nascidos pré-termo ou baixo peso. Em estatísticas americanas, verificou-se aumento de nascimentos múltiplos, ocorrendo em 3,2\% de todas as gestações em 2001 12. Em Londrina, houve aumento de nascimentos pro-

Classificação dos óbitos neonatais de acordo com critérios de evitabilidade.

\begin{tabular}{|c|c|c|c|c|c|c|}
\hline \multirow[t]{2}{*}{ Critério } & \multicolumn{2}{|c|}{1994} & \multicolumn{2}{|c|}{1999} & \multicolumn{2}{|c|}{2002} \\
\hline & $\mathrm{n}$ & $\%$ & $\mathrm{n}$ & $\%$ & $\mathrm{n}$ & $\%$ \\
\hline \multicolumn{7}{|l|}{ I - Evitáveis por } \\
\hline Adequado controle da gravidez & 38 & 45,2 & 36 & 49,0 & 24 & 54,5 \\
\hline Adequada atenção ao parto & 5 & 6,0 & 9 & 12,0 & 3 & 6,8 \\
\hline Diagnóstico e tratamento precoces & 19 & 22,6 & 9 & 12,0 & 3 & 6,8 \\
\hline Parcialmente reduzíveis & 5 & 6,0 & 2 & 2,7 & 2 & 4,5 \\
\hline II - Não evitáveis & 15 & 17,8 & 18 & 24,3 & 12 & 27,2 \\
\hline III - Mal definidas & 2 & 2,4 & & & & \\
\hline Total & 84 & 100,0 & 74 & 100,0 & 44 & 100,0 \\
\hline
\end{tabular}

Fonte: Fundação Sistema Estadual de Análise de Dados 6. 
venientes de gestações múltiplas, principalmente trigêmeos e quadrigêmeos em 2002, associado com o uso de medicações indutoras de ovulação e técnicas de reprodução assistida, como fertilização in vitro.

$\mathrm{O}$ índice de cesariana, em Londrina, é elevado, pois a Federação Internacional de Ginecologia e Obstetrícia recomenda que esse índice não deve ultrapassar 20\% 13. Em 2002, 26\% dos nascimentos nos Estados Unidos foram por cesariana 14. Em 1994, a mortalidade neonatal foi proporcionalmente maior entre as crianças nascidas por cesariana $(63 \%$ vs. $37 \%$ partos normais). Fiori et al. 15, em análise semelhante, referem que é possível que isso tenha ocorrido porque muitos recém-nascidos de baixo peso, que apresentam maior risco de óbito, nascem por meio de operações cesarianas, muitas vezes, indicadas por risco materno e fetal. Entretanto, na análise de 1999 e 2002, esse resultado não se repetiu.

A maior parte dos óbitos neonatais ocorreu nos primeiros seis dias de vida, principalmente nas primeiras 24 horas, evidenciando a estreita relação entre os óbitos e a assistência de saúde às gestantes. Uma estratégia importante para o controle é o reconhecimento de risco, com a provisão do cuidado apropriado e resolutivo à gestante e à criança, com sistematização das condutas.

A proporção de baixo peso em Londrina no ano de 2002 é semelhante a dos Estados Unidos $(7,8 \%) 14$. No Brasil, está em torno de $11 \%$, levando-se em consideração a variabilidade regional, com as maiores taxas nas regiões Norte e Nordeste. Em alguns países subdesenvolvidos como Moçambique, Bangladesh e Índia, o índice de baixo peso corresponde a $20 \%, 50 \%$ e $33 \%$, respectivamente. Em países desenvolvidos, como Suécia e Noruega, por exemplo, o percentual é de $5 \%$ e $4 \%$, respectivamente 8 .

Quando se compara o coeficiente de mortalidade neonatal em diferentes faixas de peso, verifica-se que, em Londrina, em 2002, o coeficiente para recém-nascidos com peso ao nascer de $2.500 \mathrm{~g}$ ou mais é de 1,3 óbitos por mil nascidos vivos. $\mathrm{O}$ risco de óbito nesse mesmo ano foi 12 vezes maior para recém-nascidos que pesavam entre 1.500 e $2.499 \mathrm{~g}$ e 200 vezes maior para recém-nascidos com peso menor que $1.500 \mathrm{~g}$ ao nascimento.

Nos países desenvolvidos, a mortalidade dos recém-nascidos de muito baixo peso (menores de $1.500 \mathrm{~g}$ ) vem diminuindo progressivamente nos últimos anos. Estudo realizado em 14 UTI neonatais americanas, nos anos de 1995 e 1996, mostrou sobrevida de $84 \%$ dos recém-nascidos com peso ao nascer entre 500 e $1.500 \mathrm{~g} 16$.
Em estudo de Victora \& Barros 17, a melhoria do peso ao nascer poderia levar a uma redução de $8 \%$ na mortalidade infantil no país, mas a eficácia das intervenções disponíveis é muito baixa. O baixo peso é determinado pela duração da gestação e/ou a taxa de crescimento intra-uterino. Portanto, é uma combinação de fatores diferentes que requerem intervenções distintas. Uma intervenção eficaz para reduzir o parto prematuro é a detecção e tratamento da bacteriúria assintomática na gestação. Estratégias como melhorar a nutrição materna e reduzir o fumo na gestação não mostraram mudanças significantes na mortalidade infantil 18.

$\mathrm{Na}$ análise das causas básicas de morte neonatal, além de interpretar os resultados encontrados, é importante compará-los com dados de outros serviços e populações. No entanto, muitas vezes, é difícil a interpretação de diferenças encontradas devido à falta de critérios e padronização na classificação dessas causas.

Em muitos trabalhos, as causas de morte em recém-nascidos são referidas como problemas clínicos do recém-nascido, como, por exemplo, síndrome do desconforto respiratório neonatal e prematuridade, mas é importante definir a causa básica que desencadeou todo o processo, geralmente relacionado a problemas maternos e da gravidez 19 .

Este estudo priorizou a classificação de acordo com a CID-10 5, e como, em Londrina, cada óbito infantil é considerado um "evento sentinela”, há investigação e revisão de todo o processo que culminou no óbito da criança, desde a gestação, o que permite fidedignidade nos dados.

Dentre as causas predominantes para óbitos neonatais estão: as complicações devido a problemas maternos e complicações da gestação que afetam o feto ou o recém-nascido; problemas devido à gestação de curta duração e baixo peso; e as malformações congênitas e anormalidades cromossômicas, sendo que essa última é a causa predominante em países desenvolvidos 12,14.

A mortalidade por anomalias congênitas é pouco alterada por condições sócio-econômicas e pelos avanços tecnológicos 12 . Ao analisar estatísticas de mortalidade por anomalias congênitas, deve-se considerar dois aspectos importantes: o primeiro refere-se a países onde o aborto é legalizado e, conseqüentemente, há interrupção da gestação na maioria das vezes em que há diagnóstico antenatal de malformação congênita, diminuindo o coeficiente de mortalidade neonatal 20; e o segundo aspecto refere-se à insuficiência de diagnósticos em 
óbitos precoces, levando à falsa interpretação de baixa incidência de anomalias congênitas.

A proporção de óbitos devido a malformações congênitas, em Londrina, está aumentando, e isso pode ser explicado pela melhora na assistência pré e perinatal, pois, ao diminuir o número de mortes por causas evitáveis, as malformações assumem a participação como causadoras de óbito neonatal.

É interessante que, neste estudo, a asfixia perinatal não teve participação como causa básica de óbito e foi classificada como fator associado às mortes. $\mathrm{O}$ fato é que as crianças que apresentaram asfixia ao nascimento pertenciam a grupos que tinham outras causas básicas que eram fatores de risco para asfixia. As evidências demonstram que a padronização e o treinamento em reanimação neonatal contribuem, significativamente, para a redução da mortalidade neonatal 21.

É importante ressaltar também que não houve óbito cuja causa básica foi síndrome do desconforto respiratório neonatal, pois, de acordo com os critérios do CID-10 5, a síndrome do desconforto respiratório neonatal é conseqüência da prematuridade, e esta, por sua vez, tem como causa básica fatores maternos ou problemas na gestação, que são o alvo da intervenção para evitar a prematuridade.

Em relação aos óbitos por síndrome da aspiração de mecônio (incluído em transtornos respiratórios - P20-29), verificou-se um decréscimo que pode ser justificado por melhor assistência ao recém-nascido em sala de parto, principalmente devido aos programas de treinamento em reanimação neonatal.

A classificação de infecção como causa básica de óbito deve ser muito criteriosa, pois ela está mais associada como causa imediata do óbito 22. É uma intercorrência freqüente e fator de grande morbidade, principalmente nos recém-nascidos de muito baixo peso que permanecem internados por período prolongado em UTI. À medida que os óbitos são mais bem investigados, geralmente, a infecção torna-se causa associada do óbito.

O número de mortes por causa desconhecida ou não especificada depende muito do empenho destinado ao esclarecimento dos reais fatores envolvidos com o óbito do paciente 22 .

O conceito de evitabilidade dos óbitos, seja por razões sócio-econômicas ou por políticas públicas de saúde, vem sendo bastante discutido. A classificação da Fundação SEADE baseiase no conhecimento técnico-científico existente para evitar o aparecimento de determinadas doenças ou controlá-las, independente do fato de as tecnologias estarem ou não acessíveis para a maioria da população naquele momento 23 .
Considerando as malformações congênitas como morte neonatal inevitável, conclui-se que os outros óbitos, ou seja, $82 \%, 76 \%$ e $73 \%$ dos óbitos em 1994, 1999 e 2002, respectivamente, poderiam não ter ocorrido. O objetivo da atenção perinatal é que todo feto que chegue à maturidade, sem malformação, deve sobreviver, e, atualmente, até mesmo os limites de viabilidade têm evoluído, com aumento da chance de sobrevida de prematuros cada vez menores 16 .

O decréscimo na mortalidade neonatal neste estudo pode ser atribuído à melhoria no cuidado aos recém-nascidos prematuros e de baixo peso, e não em decorrência de prevenção à prematuridade, pois o índice de prematuridade em Londrina aumentou nesses anos estudados.

\section{Conclusão}

Recomenda-se, aos serviços de saúde, abordar os óbitos neonatais como "evento sentinela", conceito proposto por Rutstein et al. 18 que definem o óbito infantil como algo que não deve ocorrer se o sistema de saúde funcionar adequadamente, ou seja, a cada óbito neonatal deve haver investigação detalhada para detectar se gestante e recém-nascido receberam assistência adequada ou se ocorreram falhas no processo de atendimento.

É muito importante definir a causa básica do óbito de acordo com o evento inicial que desencadeou todo o processo, na maioria das vezes, conseqüência de causas maternas ou da gestação, e, dessa forma, atuar de modo preventivo para evitar o óbito.

Considerando que a maior porcentagem de óbitos está relacionada com eventos que levam à prematuridade e ao baixo peso, a adequada assistência pré-natal tem papel fundamental para redução desses índices, garantindo às gestantes a realização de pré-natal, não só preocupando-se com a freqüência das consultas, mas também com a qualidade da atenção, incluindo treinamento da equipe, instituição de protocolos clínicos, utilização correta de critérios de gestação de risco e a garantia de referência para atendimento especializado quando da identificação de alto risco.

São prioritários, também, a adequada assistência ao parto para reduzir o número de cesarianas e o treinamento da equipe em reanimação do recém-nascido na sala de parto para prevenção de asfixia perinatal, aspiração de mecônio, entre outros. Os investimentos em qualidade de serviço em nível terciário com capacitação de pessoal e implementação de UTI 
neonatal são fundamentais para a adequada assistência ao recém-nascido.

É importante ressaltar que o sistema de investigação de óbitos, que detecta possíveis falhas na atenção materno-infantil, é prioritário para o conhecimento correto das causas básicas de óbito e conseqüentemente para o planejamento das ações de saúde. O Comitê de Prevenção à Mortalidade Infantil é uma estratégia recomendada pelo Ministério da Saúde 24, importante para a melhoria dos registros vitais e para a compreensão das circunstâncias de ocor- rência dos óbitos. Além disso, realiza ações educativas e sensibilizadoras, divulgando relatórios para as instituições envolvidas, desde a unidade básica que acompanhou o pré-natal até o hospital que atendeu a gestante e o recém-nascido. É uma estrutura multiprofissional, tem sido uma experiência bem-sucedida em Londrina, que pode servir de referência para os locais que ainda não operam a vigilância dos óbitos e ainda é um reforço que pode apoiar as iniciativas já existentes.

\section{Resumo}

Apesar dos avanços tecnológicos nas últimas décadas, a mortalidade neonatal é responsável por mais de $2 / 3$ dos óbitos infantis, principalmente em regiões onde o coeficiente de mortalidade infantil é baixo. O objetivo deste estudo é analisar os óbitos neonatais do Município de Londrina, Paraná, Brasil, em três períodos, iniciando por 1994 - ano de implantação das UTIs pediátrica e neonatal no município. Foram utilizados dados do SINASC e análise individualizada de cada declaração de óbito neonatal dos anos de 1994, $1999 e$ 2002. Verificou-se redução do número de nascimentos no município, o índice de baixo peso aumentou de 7,7 para 8,8\%, e o de prematuridade aumentou de 6,3 para $8,4 \%$. Houve aumento de gestações múltiplas. A taxa de cesariana variou de 48 a 52\%. O coeficiente de mortalidade neonatal declinou de 10,1 para 6,4 por mil nascidos vivos. A maioria dos óbitos são evitáveis, principalmente, por adequada atenção na gravidez. Conclui-se que está ocorrendo uma melhora progressiva na assistência ao recém-nascido, e isso é um bom indicador das ações de saúde do município.

Mortalidade Infantil; Peso ao Nascer; Unidades de Terapia Intensiva Neonatal; Unidades de Terapia Intensiva Pediátrica

\section{Colaboradores}

L. S. L. Ferrari participou na revisão de literatura, coleta de dados, análise dos resultados e redação do artigo final. A. S. J. Brito colaborou na análise dos resultados e revisão do artigo final. A. B. R. Carvalho contribuiu na revisão do artigo final. M. R. C. Gonzáles participou da coleta de dados.

\section{Agradecimentos}

À professora Tiemi Matsuo pela análise estatística e revisão das tabelas. 


\section{Referências}

1. Barros FC, Victora CG, organizadores. Epidemiologia da saúde infantil: um manual para diagnósticos comunitários. São Paulo: Editora Hucitec; 1991.

2. Laurenti R, Mello-Jorge MHP, Leibrão ML, Gotlieb SLD. Estatísticas da saúde. São Paulo: EPU/Edusp; 1987.

3. Secretaria Municipal de Saúde de Londrina. Estatísticas vitais. Londrina: Diretoria de Informações em Saúde, Secretaria Municipal de Saúde de Londrina; 2003.

4. Ferrari LSL. Estudo dos recém-nascidos vivos e da mortalidade neonatal no Município de Londrina, no ano de 1994 [Dissertação de Mestrado]. Londrina: Universidade Estadual de Londrina; 1996.

5. Organização Mundial da Saúde. Classificação estatística de doenças e problemas relacionados à saúde - 10a revisão. São Paulo: Edusp; 1996.

6. Fundação Sistema Estadual de Análise de Dados. Classificação da mortalidade infantil neonatal segundo grupos de causas de morte. São Paulo: Fundação Sistema Estadual de Análise de Dados; 1998.

7. Ministério da Saúde. Indicadores e dados básicos. http://www.datasus.gov.br (acessado em 14/Dez/ 2004).

8. Fundo das Nações Unidas para a Infância. Situação mundial da infância: 2003. Brasília: Fundo das Nações Unidas para a Infância; 2003.

9. Buehler JW, Hogue CJ, Zaro SM. Postponing or preventing deaths? Trends in infant survival, Georgia, 1974 through 1981. JAMA 1985; 253:3564-7.

10. Almeida SDM, Barros MBA. Atenção à saúde e mortalidade neonatal: estudo caso-controle realizado em Campinas, SP. Rev Bras Epidemiol 2004; 7:22-35.

11. Almeida MF, Novaes HMD, Alencar GP, Rodrigues LC. Mortalidade neonatal no Município de São Paulo: influência do peso ao nascer e de fatores sócio-demográficos e assistenciais. Rev Bras Epidemiol 2002; 5:93-107.

12. Kochanek KD, Martin JA. Supplemental analyses of recent trends in infant mortality. http: / / www. cdc.gov/nchs (acessado em 14/Dez/2004).

13. Gonzales MRC. Parto normal e cesárea: estudo epidemiológico, Londrina-1996 [Dissertação de Mestrado]. Londrina: Universidade Estadual de Londrina; 1997.
14. Arias E, MacDorman MF, Strabino DM, Guyer B. Annual summary of vital statistics - 2002. Pediatrics 2003; 112 (6 Pt 1):1215-30.

15. Fiori RM, Fiori HH, Hentschel H. Mortalidade perinatal no Rio Grande do Sul: estudo colaborativo de 16 maternidades. J Pediatr (Rio de J) 1989; 65:72-85.

16. Lemons JA, Bauer CR, Oh W, Korones SB, Papile LA, Stoll BJ, et al. Very low birth weight outcomes of the National Institute of Child health and human development neonatal research network, January 1995 through December 1996. NICHD Neonatal Research Network. Pediatrics 2001; 107:E1.

17. Victora CG, Barros FC. Infant mortality due to perinatal causes in Brazil: trends, regional patterns and possible interventions. São Paulo Med J 2001; 119:33-42.

18. Rutstein DD, Berenberg W, Chalmers TC, Child CG, Fishman AP, Perrin EB. Measuring the quality of medical care: a clinical method. N Engl J Med 1976; 294:582-8.

19. Carvalho ML, Silver LD. Confiabilidade da declaração da causa básica de óbitos neonatais: implicações para o estudo da mortalidade prevenível. Rev Saúde Pública 1995; 29:342-8.

20. Donoso-Silva E. Salud pública perinatal. Bol Esc Méd 1993; 22:81-4.

21. Sociedade Brasileira de Pediatria. Manual de reanimação neonatal. São Paulo: Universidade Federal de São Paulo; 2000.

22. Brenelli MAA, Altemani AM, Martins Filho J. Causas básicas de morte neonatal. J Pediatr (Rio de J) 1992; 68:305-11.

23. Vidal SA, Frias PG, Barreto FMP, Vanderlei LCM, Felisberto E. Óbitos infantis evitáveis em hospital de referência estadual do Nordeste brasileiro. Rev Bras Saúde Mater Infant 2003; 3:281-9.

24. Ministério da Saúde. Manual dos comitês de prevenção do óbito infantil e fetal. Brasília: Ministério da Saúde; 2004.

Recebido em 22/Dez/2004

Versão final reapresentada em 02/Jul/2005

Aprovado em 22/Ago/2005 